\title{
The effect of aquatic training on static and semi-dynamic balance of patients with chronic ischemic stroke: A randomized clinical trial
}

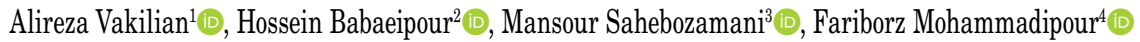 \\ 'Department of Neurology and Non Communicable Diseases Research Center, Faculty of Medicine, Rafsanjan University of Medical Sciences, Rafsanjan, Iran \\ ${ }^{2}$ Department of Sport Injuries and Corrective Trainings, Faculty of Literature and Humanity Sciences, Vali-e-Asr University of Rafsanjan, Rafsanjan, Iran \\ ${ }^{3}$ Department of Sport Injuries and Corrective Trainings, Faculty of Sport Sciences, Shahid Bahonar University of Kerman, Kerman, Iran \\ ${ }^{4}$ Department of Sport Biomechanics, Faculty of Sport Sciences, Shahid Bahonar University of Kerman, Kerman, Iran
}

Received: October 17, 2019 Accepted: May 05, 2020 Published online: September 01, 2021

\begin{abstract}
Objectives: This study aims to investigate the effect of six-week aquatic exercise on the static and semi-dynamic balance of male patients with chronic ischemic stroke (CIS).

Patients and methods: This randomized clinical trial (RCT) included a total of 36 male CIS patients (mean age $60.2 \pm 6.7$ years; range, 40 to 70 years) between January 2015 and January 2017. The patients were randomly divided into three groups consisting of 12 patients in each group: (i) exercising in shallow water, (ii) exercising in deep water, and (iii) control group. Training sessions were held three days a week for six weeks, and the control group did not participate in these sessions. The balance of the patients was checked using the Biodex balance system in two stages.

Results: There was no significant difference between the two intervention groups (shallow and deep) in terms of the balance assessment post-test $(\mathrm{p}>0.05)$, yet there was a statistically significant difference between the two intervention groups (shallow and deep) with the control group in terms of the semi-dynamic balance (total) score, $(\mathrm{p}<0.05)$; the semi-dynamic balance (total) of the two intervention groups (shallow and deep) was higher than that of the control group $(\mathrm{p}<0.05)$.

Conclusion: Our study results indicate that exercise in both depths is a suitable solution to improve balance, particularly semi-dynamic balance (total) in male CIS patients. Accordingly, aquatic training has a positive effect on balance in male stroke patients and we can prescribe this protocol as a useful remedy for these patients.
\end{abstract}

Keywords: Aquatic training, chronic ischemic stroke, semi-dynamic balance, static balance.

Stroke is the most prevalent disabling disorder of nervous system in adults, which causes significant challenges in rehabilitation medicine. Falling and balance disturbances are major stroke challenges. ${ }^{[1]}$ Falling due to reduction of static and dynamic balance is a major problem. Different studies have shown that dynamic balance is affected more than static balance $(\mathrm{SB})$ by the disease process. ${ }^{[2]}$ Currently, many rehabilitation techniques are utilized to resolve disability and balance disorders due to stroke. Hydrotherapy has been shown to enhance the immunity system, prevent falling, support pressure on musculoskeletal system, and activate the balance responses; therefore, it is a method of improving outcome. ${ }^{[3]}$ In addition, physical and supportive characteristics of viscosity, volumetric mass, floating and hydrostatic pressure provide conditions which improve muscle functions. ${ }^{[4]}$

Making changes to aquatic field by creating turbulent conditions and providing a new field for balance movement training help specialists to come up with different therapeutic solutions. ${ }^{[5]}$ Recent

Corresponding author: Hossein Babaeipour, PhD. Department of Sport Injuries and Corrective Trainings, Faculty of Literature and Humanity Sciences, Vali-e-Asr University of Rafsanjan, 7718897111 Rafsanjan, Iran.

e-mail: clinical.research85@gmail.com

Cite this article as:

Vakilian A, Babaeipour H, Sahebozamani M, Mohammadipour F. The effect of aquatic training on static and semi-dynamic balance of patients with chronic ischemic stroke: A randomized clinical trial. Turk J Phys Med Rehab 2021;67(3):315-321. 
researches have demonstrated that special exercises in the water improve mobility in the patients with disabling neurological diseases. ${ }^{[6]}$ In two other previous studies, two issues were concluded; the first one was a better sufficiency of training in the water compared to landscape to better the balance in older cases, ${ }^{[7]}$ and the second one indicated a significant improvement regarding balance following a 12-week training in the water in older cases. ${ }^{[8]}$ In another study on exercise in the water, an eight-week program resulted in better muscle performance and cardiac output as well as balanced gait among patients with post-stroke complications. ${ }^{[9]}$

On the other hand, a review of aquatic training showed the efficacy of this type of training in improving gait and speed in patients with stroke or other neurological disorders. ${ }^{[10]}$ The stroke rehabilitation program may face barriers, while the exercises are done out of water in account of lack of balance, higher risk of falls, and the fear of disability in patients. ${ }^{[1]}$ The review of the literature represented evidence of enhanced physical capabilities, increased balance, agility, spatial understanding of the body response rate velocity, increased power of muscles, balanced gait, higher range of motion, and psychological well-being in patients with stroke following aquatic exercise training. ${ }^{[1,4,5,9,10]}$ In the literature, there are no studies on balance (static and semi-dynamic) changes following an aquatic training program, particularly at two different depths in patients with chronic ischemic stroke (CIS) using the Biodex Balance System (BBB; Biodex Medical Systems Inc., NY, USA) in two stages. In the present study, we aimed to evaluate the effects of a six-week aquatic training on the SB and semi-dynamic balance (SDB) of male patients with CIS.

\section{PATIENTS AND METHODS}

This randomized clinical trial included male patients with CIS who were referred to the Ali-Ibn Abitalib Hospital between January 2015 and January 2017. Thirty-six male patients with CIS (mean age $60.2 \pm 6.7$ years; range, 40 to 70 years) were randomly divided into three groups of 12 individuals in each group, consisting of two experimental groups based on the water depth in the training program, and a control group by a team of Neurology Department and Department of Sport Injuries and Corrective Trainings (Figure 1). The patients in the intervention

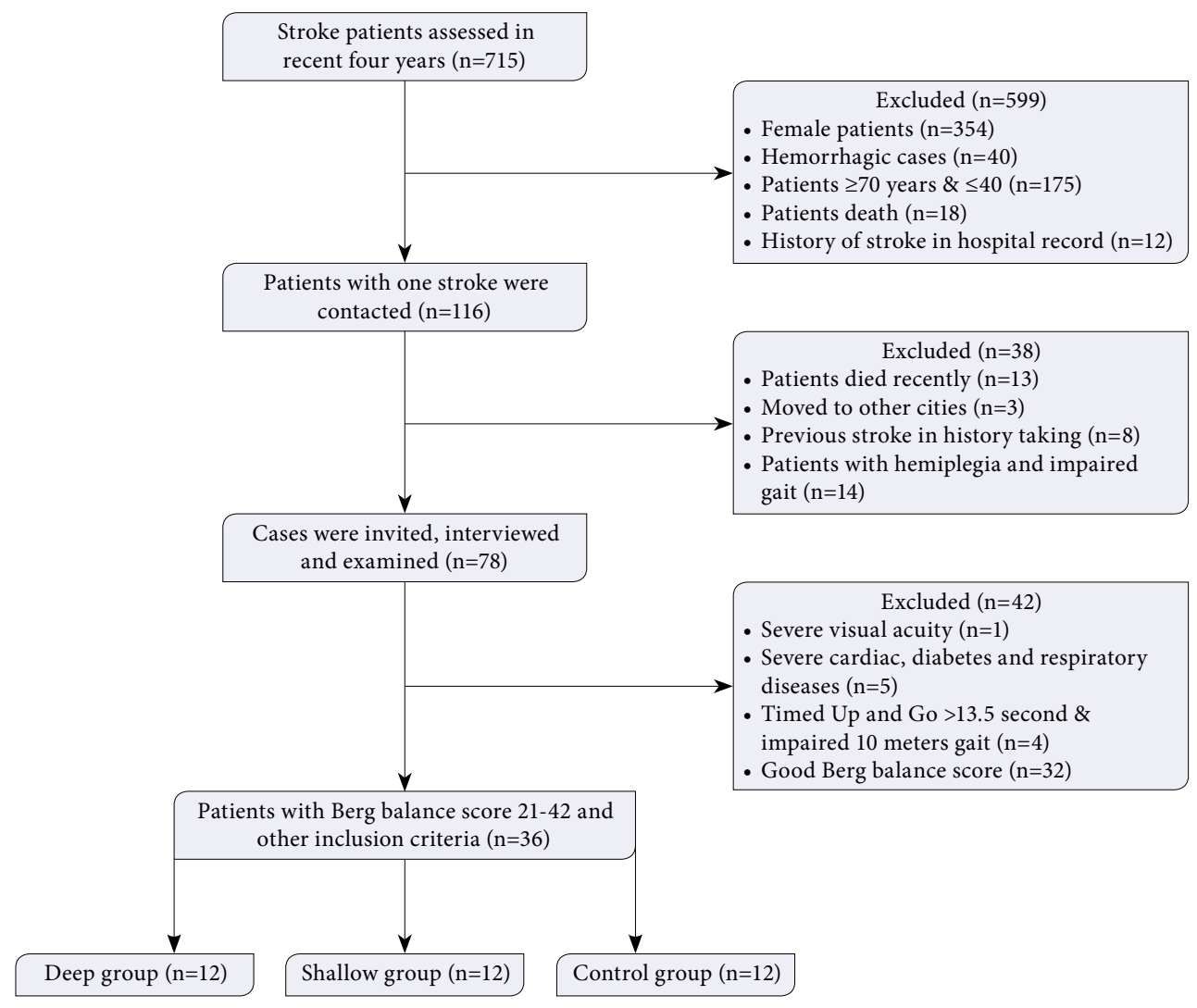

Figure 1. Study flowchart. 
groups received a six-week aquatic training program including three basic components weekly, while the controls did not receive any aquatic training. All the participants were assessed for their balance employing the BBS in two stages. Inclusion criteria were as follows: aged 40 to 70 years and having experienced their first CIS, having a muscle strength of three-fifths to four-fifths, minimum six months and maximum two years of time from the stroke incident, those who were interested in participating in the study, those who obtained a Berg Balance Scale score of 21-44, those who could walk 10 meters without assistance, being able to stand without help at least for $5 \mathrm{~min}$, and those who did not participate in any periodic physical activity or receive recent physiotherapy. Exclusion criteria were as follows: patients with certain diseases such as uncontrolled systolic hypertension during exercise or who missed more than two sessions of the training program.

Minimization was utilized as an alternative approach for ensuring excellent balance among the three groups and this method of allocation minimized inequality across multiple factors. ${ }^{[10]}$ All of the three study groups were matched for age range and balance to minimize any interference with the statistical results due to heterogeneity of the study groups without blinding. Therefore, the participants were classified into two groups, according to the two inclusion criteria of the study, the age range of 40 to 70 years and a Berg Balance Scale score range of 21 to 44 . A written informed consent was obtained from each participant. The study protocol was approved by the Ethics Committee of Rafsanjan University of Medical Sciences (No. REC.1396.207). The study was registered at the Iranian Registry of Clinical Trials (No. CT20180513039626N2) and was conducted in accordance with the principles of the Declaration of Helsinki.

Two other parts of the study, regarding the patients' quality of life, results of the Berg Balance Scale, and Timed Up and Go test were previously published. ${ }^{[1,12]}$

\section{Aquatic training sessions}

Both of the study groups received aquatic training three 30-min sessions per week for a total of six weeks, while the water temperature was 34 to $36^{\circ} \mathrm{C}$ and the air temperature was set to $24^{\circ} \mathrm{C} \cdot{ }^{[13]}$ According to the principle of scientific practice, matching the type and pattern of movements in each session was done in both of them. Exercise in the first session began with

\section{TABLE 1}

Method of implementation of aquatic training

\begin{tabular}{|c|c|c|c|c|c|c|c|c|}
\hline \multirow[b]{2}{*}{ Week } & \multirow[b]{2}{*}{ Session } & \multirow[b]{2}{*}{ Number of exercise } & \multirow[b]{2}{*}{ Set } & \multirow[b]{2}{*}{ Repeat } & \multicolumn{3}{|c|}{ Activity time $60 \mathrm{~min}$} & \multirow[b]{2}{*}{ Rest minutes } \\
\hline & & & & & Exercise & Warming & Cooling & \\
\hline \multirow{3}{*}{ First } & 1 & 9 & 1 & 3 & 50 & 5 & 5 & 15 \\
\hline & 2 & 9 & 1 & 4 & 50 & 5 & 5 & 14 \\
\hline & 3 & 9 & 1 & 6 & 50 & 5 & 5 & 13 \\
\hline \multirow{3}{*}{ Second } & 4 & 9 & 1 & 8 & 50 & 5 & 5 & 12 \\
\hline & 5 & 9 & 1 & 10 & 50 & 5 & 5 & 11 \\
\hline & 6 & 9 & 1 & 10 & 50 & 5 & 5 & 11 \\
\hline \multirow{3}{*}{ Third } & 7 & 9 & 2 & 6 & 50 & 5 & 5 & 10 \\
\hline & 8 & 9 & 2 & 6 & 50 & 5 & 5 & 10 \\
\hline & 9 & 9 & 2 & 8 & 50 & 5 & 5 & 9 \\
\hline \multirow{3}{*}{ Fourth } & 10 & 9 & 2 & 8 & 50 & 5 & 5 & 9 \\
\hline & 11 & 9 & 2 & 10 & 50 & 5 & 5 & 8 \\
\hline & 12 & 9 & 2 & 10 & 50 & 5 & 5 & 8 \\
\hline \multirow{3}{*}{ Fifth } & 13 & 9 & 3 & 8 & 50 & 5 & 5 & 6 \\
\hline & 14 & 9 & 3 & 8 & 50 & 5 & 5 & 6 \\
\hline & 15 & 9 & 3 & 9 & 50 & 5 & 5 & 4 \\
\hline \multirow{3}{*}{ Sixth } & 16 & 9 & 3 & 9 & 50 & 5 & 5 & 4 \\
\hline & 17 & 9 & 3 & 10 & 50 & 5 & 5 & 3 \\
\hline & 18 & 9 & 3 & 10 & 50 & 5 & 5 & 3 \\
\hline
\end{tabular}


a three-repetition set and, finally in the final session, it ended with three 10-repetition sets to observe the principle of exercise. The patients were given a resting time of $15 \mathrm{~min}$ in the first session, which finally decreased to $3 \mathrm{~min}$ (session \#18) to increase the training load by reducing the resting time (Table 1).

For the first group (shallow group), the training was conducted in hip-deep (anterior superior iliac spine) water, whereas it was conducted in chest-deep (xiphoid process sternum) water in the second group (deep group). Throughout the exercise sessions, two lifeguards were present for patients' safety and peace of mind, according to the exercise protocol. ${ }^{[5]}$

Aquatic training included hip (flexion, extension, and rotation), lunge gaiting, backward gaiting, hip abductor stretch, tensor fasciae latae stretch, standing on one leg, sidestepping, and knee bending. ${ }^{[1]}$ The BBS was used in the present study to check the patients' balance (static and semi-dynamic). We measured the balance performance in the participants employing the BBS. The plate stiffness was adjusted using a relevant software to test postural stability on the static surface (measuring patient's SB, as well as a modified clinical test of sensory interaction in balance (CTSIB-M) on the semi-dynamic surface. ${ }^{[14]}$ Postural stability test was performed to measure postural deviations to analyze fluctuations based on four indicators including overall stability index (OSI), anterior-posterior stability index (APSI), medial-lateral stability index (MLSI), and trunk total sway (TTS). The CTSIB-M test was performed in four different positions on two legs with the following modes: open eye, tight surface-closed eye, tight surface-open eye, foam surface-closed eye, foam level.

Each test was in progress for $30 \mathrm{sec}$, including three repetitions with 30 -sec rest intervals. The aforementioned tests were performed in a quiet place to maximize patients' concentration, since any voice or noise could interfere with the correct performance of patients and distort the recorded results. Ultimately, the OSI, APSI, MLSI, TTS, and CTSIB-M at preand post-test stages were calculated for all the three groups. ${ }^{[14,15]}$

\section{Statistical analysis}

Statistical analysis was performed using the IBM SPSS for Windows version 21.0 software (IBM Corp., Armonk, NY, USA). Descriptive data were presented in mean \pm standard deviation (SD) for numeric variables and in number and percentage for categorical variables. Continuous variables were compared using one-way analysis of variance (ANOVA) across the three prespecified groups of the shallow practice, deep practice, and control. As there was more than one dependent variable including SB and SDB, multivariate analysis of covariance (MANCOVA) was employed to determine whether the means of dependent variables were equal across the three groups, while they were being statistically controlled for the effect of pretraining variable. On condition that MANCOVA showed a significant result among the groups, the least significant difference (LSD) post-hoc test was utilized to determine the differences between the means of the groups in terms of balance, which is of great significance. The Bonferroni multiple comparisons test was used to determine which means among the three means differed from the rest. The Pillai's trace was used as a test statistic in the MANCOVA. The Pillai's trace value was reported along with associated F-statistic and $p$ value. In general, small $p$ values $(<0.05)$ imply that at least one of the dependent variables has a statistically significant different mean among the studied groups. The Shapiro-Wilk test was applied to examine normal distribution of each dependent variable in the three groups. The Levene's test was used to examine homogeneity of variances across the three groups. The Box's $M$ test was also used to examine the equality of intercorrelation matrix between dependent variables across all the groups. A two-tailed $p$ value of $<0.05$ was considered statistically significant.

\section{RESULTS}

There was no significant difference in the age among the three groups $(\mathrm{p}=0.889$ ). According to our findings, there was also no significant difference among the three groups in terms of height, weight, and body mass index $(\mathrm{p}>0.05)$ (Table 2$)$. Table 3 shows the results of the mean variable balance separately in patients with stroke in pre-test and post-test intervention and control groups using ANOVA. In both groups (shallow and deep), a significant increase in the mean balance was observed compared to pre-test and post-test stages. However, this increment in the control group was not significant.

A significant difference was also observed in the results of the Pillai's trace test between the intervention and control groups in terms of the patients balance with CIS ( $\mathrm{p}=0.001$ ), indicating that in the Pillai's trace test there should be a significant difference among the three groups in terms of the balance criteria $(\mathrm{p}=0.002)$. The squared eta values implied that about $30 \%$ of the variance or difference of the scores among 


\begin{tabular}{|c|c|c|c|c|}
\hline \multicolumn{5}{|c|}{$\begin{array}{c}\text { TABLE } 2 \\
\text { Baseline demographic characteristics of male patients with chronic ischemic stroke }\end{array}$} \\
\hline & Shallow practice group & Deep practice group & Control group & \\
\hline & Mean \pm SD & Mean \pm SD & Mean \pm SD & $p$ \\
\hline Age (year) & $59.50 \pm 7.103$ & $60.17 \pm 7.383$ & $60.83 \pm 5.48$ & 0.889 \\
\hline Weight (kg) & $70.500 \pm 9.115$ & $75.833 \pm 14.622$ & $68.625 \pm 8.09$ & 0.263 \\
\hline Height $(\mathrm{cm})$ & $164.92 \pm 5.961$ & $166.67 \pm 4.60$ & $164.33 \pm 7.73$ & 0.638 \\
\hline Body mass index $\left(\mathrm{kg} / \mathrm{m}^{2}\right)$ & $25.974 \pm 3.42$ & $27.216 \pm 4.502$ & $25.433 \pm 2.72$ & 0.473 \\
\hline
\end{tabular}

\begin{tabular}{|c|c|c|c|c|}
\hline \multicolumn{5}{|c|}{$\begin{array}{c}\text { TABLE } 3 \\
\text { Static balance and semi-dynamic balance test }\end{array}$} \\
\hline & Shallow practice group & Deep practice group & Control group & \multirow[b]{2}{*}{$p^{*}$} \\
\hline & Mean \pm SD & Mean \pm SD & Mean \pm SD & \\
\hline \multicolumn{5}{|l|}{ Static balance } \\
\hline Pre-training & $4.5 \pm 3.2$ & $3.6 \pm 2.2$ & $3.5 \pm 1.6$ & 0.541 \\
\hline Post-training & $2.3 \pm 1.0$ & $2.1 \pm 1.2$ & $3.5 \pm 1.9$ & 0.037 \\
\hline \multicolumn{5}{|c|}{ Semi-dynamic balance } \\
\hline Pre-training & $0.9 \pm 0.2$ & $0.8 \pm 0.3$ & $0.9 \pm 0.3$ & 0.714 \\
\hline Post-training & $0.6 \pm 0.2$ & $0.5 \pm 0.1$ & $0.9 \pm 0.2$ & 0.001 \\
\hline
\end{tabular}

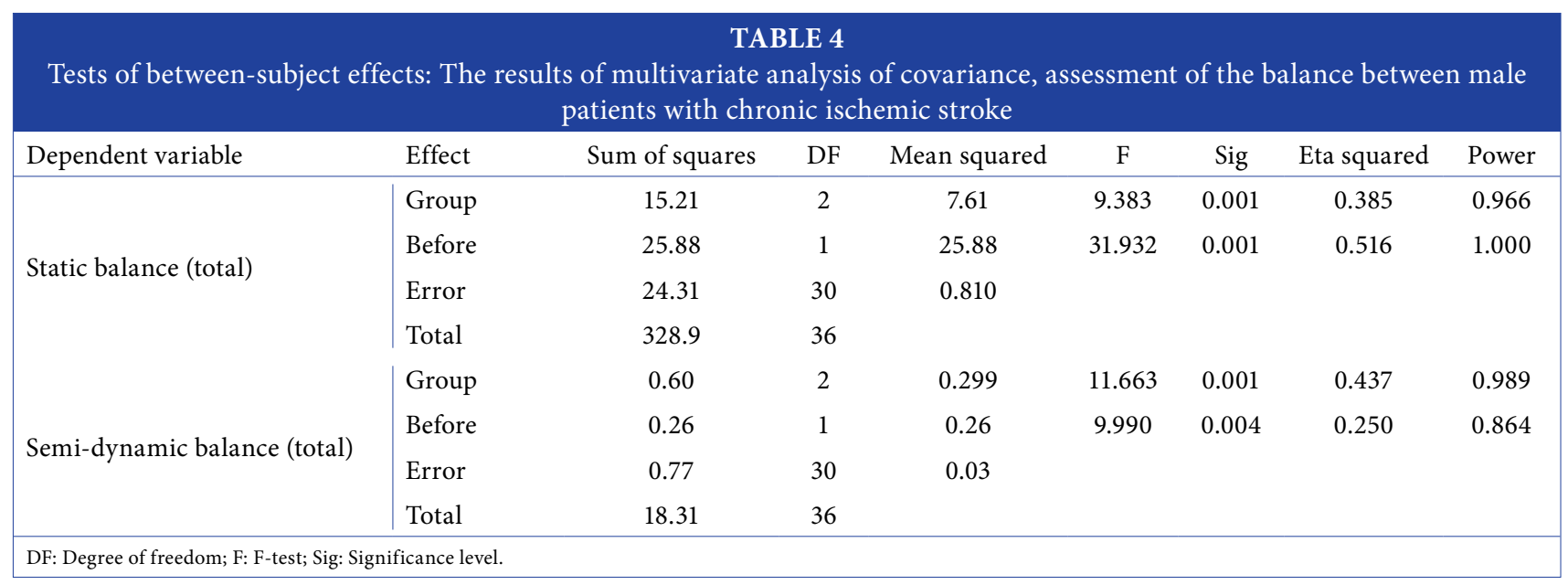

\section{TABLE 5}

Results of the least significant difference post-hoc test in the balance assessments between the two intervention (shallow and deep) groups and control group in male patients with chronic ischemic stroke

\begin{tabular}{|l|llccc|}
\hline Variable & Group A & Group B & Mean difference (A, B) & SE & $p^{*}$ \\
\hline \multirow{3}{*}{ Static balance (total) } & \multirow{2}{*}{ Shallow } & Deep & 0.1792 & 0.5777 & 0.758 \\
& & Control & 1.252 & 0.5777 & 0.038 \\
& \multirow{3}{*}{ Deep } & Control & 1.431 & 0.5777 & 0.019 \\
Semi-dynamic salance (total) & \multirow{3}{*}{ Shallow } & Deep & 0.102 & 0.07950 & 0.210 \\
& & Control & 0.2100 & 0.07950 & 0.013 \\
& \multirow{2}{*}{ SE: Standard error; ${ }^{*}$ Significant difference $\mathrm{p}<0.05 \%$. } & Control & 0.3117 & 0.07950 & 0.001 \\
\hline
\end{tabular}


the three groups in the studied variables was due to the intervention effect. The results of the MANCOVA showed significant differences in the balance results among the three groups $(\mathrm{p}<0.001)$ (Table 4$)$.

To achieve a better comprehension of the fact that after the intervention, which difference between the groups is the significant, the LSD post-hoc test was used (Table 5). Although there was no statistically significant difference between the two intervention groups (shallow and deep) in the post-test stage regarding the two balance domains ( $\mathrm{p}>0.05)$, there was still a significant difference between these two groups (shallow and deep) and the control group regarding both domains $(\mathrm{p}>0.05)$.

\section{DISCUSSION}

The results of the current study proved that the effect of a six-week aquatic training was significant on both the static and the SDB in male patients with CIS. Moreover, this study shed light into the fact that the aquatic therapy could positively affect the balance of male patients with CIS in both intervention groups compared to the control group. These findings are consistent with those of Zhu et al., ${ }^{[13]}$ Duffy ${ }^{[16]}$ Motta, ${ }^{[17]}$ Daniel et al., ${ }^{[18]}$ and Kamali et al. ${ }^{[19]}$

Previous studies have also evaluated the efficacy of difference depths for water balance test in patients with CIS and have shown the efficacy of deep-water exercise in balance improvement of patients. ${ }^{[17,19]}$ According to the present study, there was no significant difference between the intervention groups in terms of the post-test results in both depths. One of the possible reasons for these similar results could be the utilization of the six-week protocol. This finding might be far more different in a longer period of time. Balance improvement in the shallow water might be the result of the kinetic chain activity and engagement of the lower limb joints, whereas improvement in the deep water could be the result of water pressure and unstable environment. ${ }^{[17]}$

There was no significant difference in the post-test stage between the two intervention groups. However, there was a significant difference between the two intervention groups and the control group in terms of the total SDB of the post-test results. One of the possible reasons for the imbalance in most of the current study participants was their low motivation for stand-up challenges, compared to walk challenges, as their fluctuations were significantly higher in standing than walking. Therefore, the balance disruptive force of water can provide a good environment for balance activities and challenging the systems involved in balancing and improving the semi-dynamic stability. Regarding other possible reasons, the environmental conditions allow the patient to perform a wide range of movements without fear of falling or getting hurt. Additionally, the water conditions expose patients to a permanent resistance to movement, which could lead to loss of balance and, consequently, the patient's effort to restore balance. On the other hand, the viscosity of water increases neural adaptation, which could enhance the stability. ${ }^{[20,21]}$ In other words, the results of the current study indicated that a six-week exercise program had a significant effect on the improvement of balance in male patients with CIS, which is consistent with the findings of previous studies that confirmed that a program of aquatic training would help male patients with CIS to improve their mobility. ${ }^{[5,22]}$

Furthermore, Zhu et al. ${ }^{[13]}$ reported that marine training improved mobility, static, and dynamic balance in patients with CIS. In another study, an eight-week aquatic exercise on the protocol of Halliwick and Ai Chi improved postural balance in patients with chronic osteoarthritis. ${ }^{[23]}$ Santos et al. ${ }^{[5]}$ also found that CIS disabilities were improved during a 12-session hydrotherapy program, comparing the pre-test and post-test results. These findings showed that the aquatic training might be a useful technique to improve motor performance in patients with CIS. Recently, the aquatic training has remarkably drawn attention of physiotherapist and researchers to itself in view of its advantages. In addition, the buoyancy of human body in water helps such patients to move comfortably; the situation that is not possible on land helplessly. ${ }^{[24,25]}$

The small sample size and enrolling only male participants with ischemic, chronic, and hemiparetic complications were among the main limitations of the present study. Therefore, further large-scale, long-term, prospective studies are needed using the same therapeutic method among patients with hemorrhagic stroke, and chronic, debilitating neurological diseases of both sexes.

In conclusion, our study results suggest that, although aquatic training has a positive effect on balance in patients with stroke and it can even be prescribed as a remedy, the depth of water (shallow or deep) does not affect the improvement of balance. These findings enable us to extend the research on larger groups of subjects. The current study can be applied by medical practitioners, departments of rehabilitation, physiotherapists, 
clients, and even sports practitioners. Based on these results, exercise in both depths is a suitable solution to improve balance, particularly total SDB, in male patients with CIS. Water as a low-risk, safe, and effective environment can help to handle training activities for the treatment of patients with CIS and the improvement of balance in such patients.

\section{Acknowledgements}

We would like to express our thanks to Clinical Research Developmental Center of Ali-Ibn Abitaleb Hospital. We also thank all the Deputy Chairman of the research department of Rafsanjan University of Medical Sciences for the financial support and the patients who participated in this study.

\section{Declaration of conflicting interests}

The authors declared no conflicts of interest with respect to the authorship and/or publication of this article.

\section{Funding}

This project was supported financially by Vice Chancellery for Research and technology of Rafsanjan University of Medical Sciences with Grant Number (96170).

\section{REFERENCES}

1. Mackintosh SF, Hill KD, Dodd KJ, Goldie PA, Culham EG. Balance score and a history of falls in hospital predict recurrent falls in the 6 months following stroke rehabilitation. Arch Phys Med Rehabil 2006;87:1583-9.

2. Flansbjer UB, Blom J, Brogårdh $\mathrm{C}$. The reproducibility of Berg Balance Scale and the Single-leg Stance in chronic stroke and the relationship between the two tests. PM R 2012;4:165-70.

3. Yen CL, Wang RY, Liao KK, Huang CC, Yang YR. Gait training induced change in corticomotor excitability in patients with chronic stroke. Neurorehabil Neural Repair 2008;22:22-30.

4. Becker BE. Aquatic therapy: scientific foundations and clinical rehabilitation applications. PM R 2009;1:859-72.

5. Santos DG, Pegoraro AS, Abrantes CV, Jakaitis F, Gusman S, Bifulco SC. Evaluation of functional mobility of patients with stroke sequela after treatment in hydrotherapy pool using the Timed Up and Go Test. Einstein (Sao Paulo) 2011;9:302-6.

6. Cole A, Johnson J, Alford J, Hard K, Moschetti M, Fredericson M. Aquatic rehabilitation strategies. In: Becker BE, Cole AJ, editors. Comprehensive aquatic therapy. Washington: Washington State University Press; 2011. p. 56-72.

7. Douris P, Southard V, Varga C, Schauss W, Gennaro C, Reiss A. The Effect of Land and Aquatic Exercise on Balance Scores in Older Adults. Journal of Geriatric Physical Therapy 2003:26:3-6.

8. Waters D, Hale L. Do aqua-aerobics improve gait and balance in older people? A pilot study. International journal of therapy and rehabilitation 2007:14;538-43.

9. Chu KS, Eng JJ, Dawson AS, Harris JE, Ozkaplan A, Gylfadóttir S. Water-based exercise for cardiovascular fitness in people with chronic stroke: a randomized controlled trial. Arch Phys Med Rehabil 2004;85:870-4.
10. Marinho-Buzelli AR, Bonnyman AM, Verrier MC. The effects of aquatic therapy on mobility of individuals with neurological diseases: a systematic review. Clin Rehabil 2015;29:741-51.

11. Babaeipour H, Sahebozamani M, Mohammadipour F, Vakilian A. The effect of Training at different depths on the balance of chronic ischemic stroke patients. IJAEP Mazandaran 2018;7:68-78.

12. Babaeipour H, Sahebozamani M, Mohammadipour F, Vakilian AR. The effect of six weeks of aquatic training on the quality of life in patients with chronic ischemic stroke: A randomized clinical trial. J Rafsanjan Univ Med Sci 2018;17:699-714.

13. Zhu Z, Cui L, Yin M, Yu Y, Zhou X, Wang H, et al. Hydrotherapy vs. conventional land-based exercise for improving walking and balance after stroke: a randomized controlled trial. Clin Rehabil 2016;30:587-93.

14. Arnold BL, Schmitz RJ. Examination of balance measures produced by the biodex stability system. J Athl Train 1998;33:323-7.

15. Cachupe WJC, Shifflett B, Kahanov L, Wughalter EH. Reliability of Biodex Balance System Measures. Meas Phys Educ Exerc Sci 2001:5:2;97-108.

16. Duffy K. Aquatic therapy for a patient post-stroke: A case report, Florida Gulf Coast University [Internet] 2014;10:422431. Available at: https://fgcu.digital.flvc.org/islandora/ object/fgcu\%3A27267 [Accessed: October 01, 2019]

17. Motta M. The effects of aquatic and land-based exercise on balance and gait in people post stroke. Northridge: California State University; 2013.

18. Daniel K, Wolfe CD, Busch MA, McKevitt C. What are the social consequences of stroke for working-aged adults? A systematic review. Stroke 2009;40:e431-40.

19. Kamali M, Ghasemi B, Salehi M. Effect of aquatic and landbased exercises on post- stroke postural balance of women. Feyz JKUMS 2016;20:11-23.

20. Tavakol A, Daneshjoo A, Sahebozamani M. Effect of six weeks shallow and deep water exercises on static balance and pain of girls with patellofemoral pain. J Rehab Med FALL 2016;5:111-8.

21. Suzuki M, Fujisawa H, Machida Y, Minakata S. Relationship between the Berg Balance Scale and Static Balance Test in Hemiplegic Patients with Stroke. J Phys Ther Sci 2013;25:1043-9.

22. Dziubek W, Bulińska K. The effects of aquatic exercises on physical fitness and muscle function in dialysis patients. Biomed Res Int 2015;41:31-40.

23. Zamanian F, Vesalinaseh M, Nourollahnajafabadi M, Asadysaravi S, Haghighi M. Comparison of the effects of aquatic exercise in shallow and deep water on postural control in elderly women with chronic knee osteoarthritis. Life Sci J 2012;9:5768-71.

24. Kim K, Lee DK, Kim EK. Effect of aquatic dual-task training on balance and gait in stroke patients. J Phys Ther Sci 2016;28:2044-7.

25. Donyapour H, Abedini M, Eskandarnejad M, Mohammadzade H. Effects of pilates and neurofeedback exercises on balance and cognitive disorders in chronic stroke patients. J Rehab Med 2015;4:127-37. 